\title{
Evaluating the effectiveness of a
} support programme for people with type 2 diabetes mellitus in primary care: an observational prospective study in Ecuador

\author{
Francisco Barrera-Guarderas ${ }^{1}$, Katherine De la Torre-Cisneros ${ }^{2 *}$, \\ Maria Barrionuevo-Tapia ${ }^{3}$, Carmen Cabezas-Escobar ${ }^{4}$
}

${ }^{1}$ College of Medicine, Pontifical Catholic University of Ecuador, Quito, Ecuador;

${ }^{2}$ College of Medical Sciences, State University of Southern Manabi, Jipijapa, Ecuador;

${ }^{3}$ College of Medicine, Pontifical Catholic University of Ecuador, Quito, Ecuador;

${ }^{4}$ College of Medicine, Pontifical Catholic University of Ecuador, Quito, Ecuador

*For correspondence: katherine. delatorre@unesum.edu.ec

Competing interest: The authors declare that no competing interests exist.

Received: 24 September 2019 Accepted: 24 October 2019

Published: 22 April 2020

@This article is Open Access: CC BY license (https://creativecommons.org/licenses/ by/4.0/)

Author Keywords: diabetes mellitus, primary health care, non-communicable diseases, health systems

Copyright (C) 2020, The Authors;

DOI:10.3399/

bjgpopen20X101025

\begin{abstract}
Background: The success of primary health care relies on the integration of empowered practitioners with cooperative patients regardless of socioeconomic status. Using resources efficiently would help to improve healthcare promotion and reduce complications of chronic non-communicable diseases (NCDs). The importance of network support programmes relies on the fact that they allow to accurately deliver medical care by shaping a sense of community and purpose among the patients.
\end{abstract}

Aim: To evaluate the effectiveness of a network support programme for patients with type 2 diabetes mellitus (T2DM).

Design \& setting: A centre-based observational prospective study took place in a primary care setting in Ecuador.

Method: The impact of the diabetes care programme was assessed by comparing initial and final metabolic characteristics and outcomes of 593 patients with T2DM, followed-up from April 2007 to December 2017, using paired sample t-test. Electrocardiograms (ECGs), ankle-brachial indexes (ABIs), ocular fundus, and monofilament neuropathy tests were assessed with the McNemar test to evaluate complications at the beginning and end of the study.

Results: Glycated haemoglobin ( $\mathrm{HbA} 1 \mathrm{c})$, lipid profile, and systolic blood pressure (SBP) showed statistically significant decreases between the initial measurement (IMs) and final measurements (FMs). In the FM, significantly lower $\mathrm{HbA1c}$, diastolic blood pressure (DBP), and atherogenic index were found. Despite the length of time since diagnosis, during the follow-up time, long-term micro- and macro-vascular complications, such as ocular fundus, serum creatinine, and $A B I$, remained unchanged throughout the period of active participation in this healthcare programme.

Conclusion: This study demonstrates the feasibility of reducing plasma glucose, plasma lipids, and long-term complications in patients with T2DM by implementing a network support programme, which involves the medical team and patients themselves in an environment with limited resources. 


\section{How this fits in}

There are successful medical care models worldwide; however, in Latin American countries, application of these models is difficult to fulfil owing to the lack of public policies. This study shows that a comprehensive intervention can be beneficial in the medium- and long-term prevention of diabetes complications when delivered in primary health care in developing countries.

\section{Introduction}

The prevalence of T2DM, initially considered a disease of high-income countries, has become a significant public health problem increasingly affecting low- and middle-income countries. ${ }^{1}$ It is predicted that 550 million people will have T2DM by 2030, an increase of $50 \%$ compared with current data. ${ }^{2}$ In 2017, $8 \%$ of the adult population of Central and South America was estimated to suffer from diabetes; in Ecuador, it is estimated that 5.5\% (3.5\%-8.5\%) of the population aged between $20-79$ years have $\mathrm{T}_{2} \mathrm{DM} .^{3}$

The Ecuadorian Constitution protects and implements the right to health. The National Plan for Good Living was developed as a strategy to transform the structure of health care and, therefore, improve the quality of life of the Ecuadorian population. To this end, the Comprehensive Health Care Model (Modelo de Atención Integral en Salud [MAIS]) has gradually been implemented in health centres across the country with a focus on chronic diseases. ${ }^{4}$

The Chimbacalle Medical Centre, operated by the Ministry of Public Health of Ecuador, follows the health policy guidelines regarding usual care for patients with T2DM. It consists of a general medical consultation once every 3 months, in which the patient receives general advice and medicine. However, this particular healthcare centre offers a unique additional programme that aims to improve the care of patients with NCDs, specifically patients with T2DM. Patient care was led by one internist and one nurse during the continuity of the entire health programme, and was supported by family medicine rotating-resident physicians. The diabetes care programme strengthens the participation of patients through the mobilisation of a support network called the 'Patients with Diabetes Club'. This organisational model serves to improve the quality of care mostly for people with low-middle incomes, although the service is open to every patient who might need the services provided by the health centre. ${ }^{5}$

The objective of this study was to evaluate the effectiveness of the diabetes care programme for patients with T2DM by comparing the metabolic characteristics of the patients when they entered the programme with their metabolic characteristics after taking part in the programme (April 2007 to December 2017).

\section{Method}

\section{Setting}

The Chimbacalle Medical Centre is a community healthcare centre located 2800 metres above sea level in the metropolitan area of Quito, Ecuador. It is operated by the Ministry of Public Health of Ecuador and provides free laboratory tests, complementary examinations, and medication. It offers health services for men and women with limited economic resources.

\section{Study design}

A centre-based observational prospective study was conducted to evaluate the impact of the comprehensive healthcare approach to T2DM provided by Chimbacalle Medical Centre in Quito, Ecuador.

The diabetes clinic was started to deliver a strategy to fulfil the need to provide integral attentionfocused care to the increasing number of patients with T2DM. The programme also incorporated patients' relatives that were diagnosed with T2DM through a research of glucose alterations. Moreover, this unique programme is open to all patients with T2DM, regardless of their personal characteristics. Owing to this, a third source of recruitment consists in the referral of patients (cases with low or high complexity) with a stable condition from other healthcare centres.

Owing to the growth in the number of patients, there are now educational talks every 15 days in the health centre and physical activities, such as dancing and tai chi, take place three times a week. The 


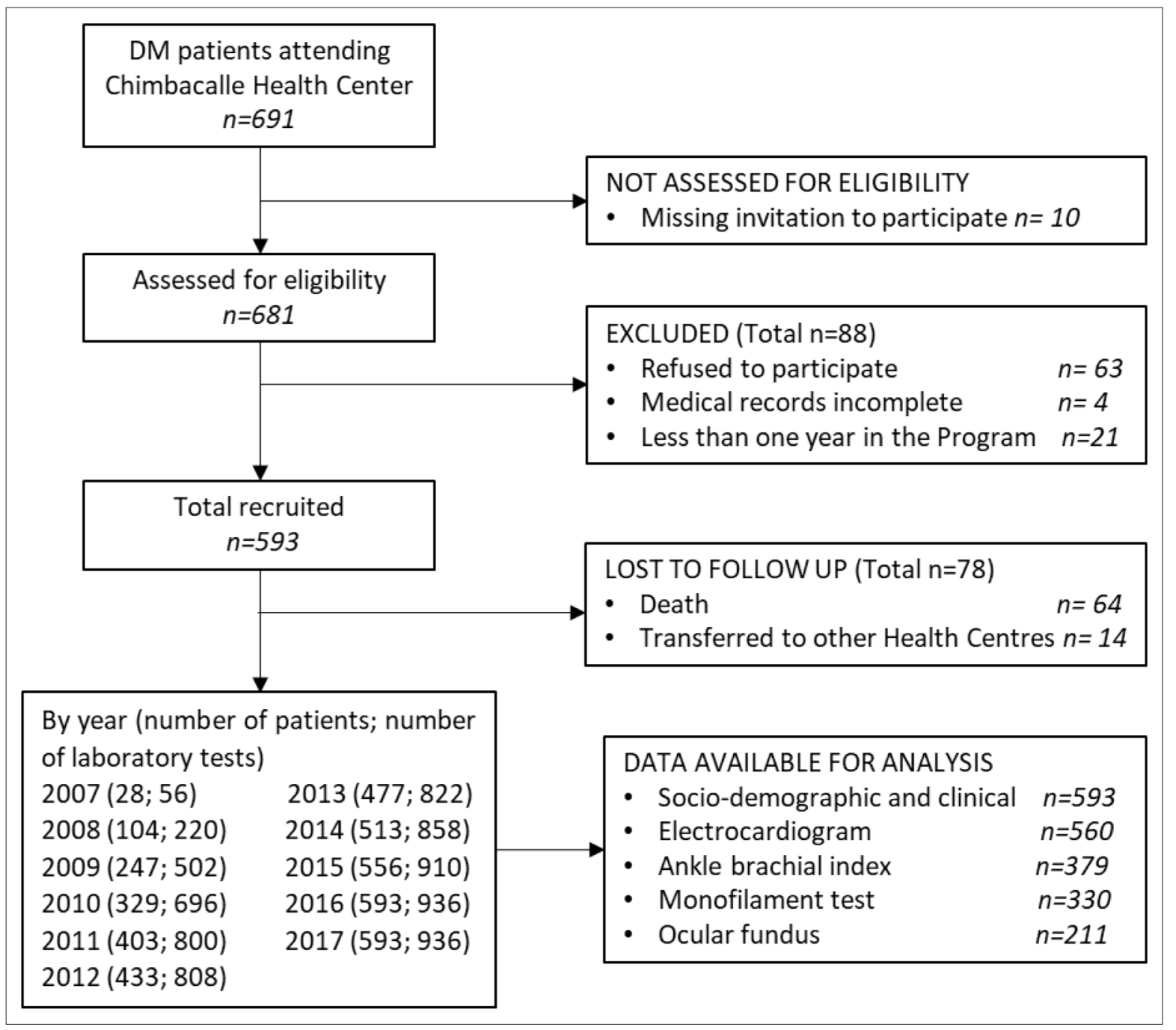

Figure 1 Flow diagram of patient recruitment and follow-up. DM = diabetes mellitus.

programme of diabetes care includes cardiovascular $r^{6,7}$ and renovascular ${ }^{8,9}$ prophylaxis, lipid-lowering medication, ${ }^{10,11}$ hypoglycants, ${ }^{12-15}$ and antihypertensives medication (Supplementary Table 1).

Study individuals had to have a record of their initial consultation, consisting of an analysis of the history of the disease, its evolution, the pillars of the treatment to be followed by the patient, and the initial indications. Patients had a week to complete individual laboratory tests, ECG, ABI, monofilament test, and oculus fundus before a second consultation. The laboratory tests included blood count, glucose, kidney profile (including albuminuria), liver profile, lipid profile, and glycosylated haemoglobin A1c. These examinations would be repeated at 4-6 months, according to the guidelines for clinical practice. ${ }^{16}$ Afterwards, fasting-glucose monitoring and daily post-prandial assessment would be performed every 2 hours for a week with a capillary glucose test, which allows the medication or insulin dose to be corrected. ${ }^{17}$ Mean plasma levels of lipid profile, $\mathrm{HbA} 1 \mathrm{c}$, urea, and creatinine were measured with a chemistry analyser (Mindray BS-200). SBP, DBP, and body mass index (BMI) were determined for each measurement stage. Atherogenic indexes were calculated using their respective formulas. ${ }^{18,19}$

Data gathered from the medical records by the medical team included sociodemographic variables and IM and FM metabolic variables in patients with between 1-10 years of treatment at the medical centre. All patients $(n=593)$ who were diagnosed or treated for T2DM at the medical centre for $\geq 1$ year and who had data available for their metabolic features by December 2017 (Figure 1) were included in the study regardless of age, sex, or other medical conditions. 
Table 1 Baseline characteristics of patients with

\begin{tabular}{lccc}
\hline Characteristics & $\begin{array}{c}\text { All, } \\
\mathbf{N}=\mathbf{5 9 3}\end{array}$ & $\begin{array}{c}\text { Female, } \\
\mathbf{n = 4 5 9}\end{array}$ & $\begin{array}{c}\text { Male, } \\
\boldsymbol{n}=134\end{array}$ \\
\hline $\begin{array}{l}\text { Mean age, } \\
\text { years (SD) }\end{array}$ & $\begin{array}{c}66.5 \\
(12.3)\end{array}$ & $66.5(12.0)$ & $66.5(13.5)$ \\
\hline $\begin{array}{l}\text { Mean age at } \\
\text { diagnosis, } \\
\text { years (SD) }\end{array}$ & $\begin{array}{c}52.7 \\
(12.2)\end{array}$ & $52.3(11.8)$ & $53.8(13.7)$ \\
\hline $\begin{array}{l}\text { Mean time of } \\
\text { disease, years } \\
\text { (SD)* }\end{array}$ & $13.8(7.9)$ & $14.2(8.0)$ & $13.0(7.4)$ \\
$\begin{array}{l}\text { Mixed race, } \\
n(\%)\end{array}$ & $588(99.2)$ & $456(77.6)$ & $132(22.4)$ \\
\hline $\begin{array}{l}\text { Hypertension, } \\
n(\%)\end{array}$ & $387(65.3)$ & $306(79.1)$ & $81(20.9)$ \\
\hline $\begin{array}{l}\text { Family history } \\
\text { of CVD, } n(\%)\end{array}$ & $343(57.8)$ & $262(76.4)$ & $81(23.6)$ \\
\hline
\end{tabular}

\begin{tabular}{lccc}
\hline Tobacco use* & & & \\
\hline $\begin{array}{l}\text { Non-smokers, } \\
n(\%)\end{array}$ & $464(78.2)$ & $404(87.1)$ & $60(12.9)$ \\
\hline $\begin{array}{l}\text { Former } \\
\text { Smokers, } n(\%)\end{array}$ & $83(14.0)$ & $40(48.2)$ & $43(51.8)$ \\
\hline $\begin{array}{l}\text { Smokers, } n(\%) \\
\text { Mean }\end{array}$ & $46(7.8)$ & $15(32.6)$ & $31(67.4)$ \\
\hline $\begin{array}{l}\text { programme } \\
\text { engagement } \\
\text { time, years } \\
(\mathrm{SD})^{*}\end{array}$ & & $5.7(2.6)$ & $4.9(2.7)$ \\
\hline
\end{tabular}

$\mathrm{CVD}=$ cardiovascular disease. $\mathrm{SD}=$ standard deviation.

*Time of disease, programme engagement time, and tobacco use were significantly different among the sexes (Mann-Whitney U-test, $\chi^{2}$ test; statistically significant $P<0.05$ ). type 2 diabetes mellitus.

Statistical analysis of the data was performed with IBM SPSS Statistics (version 23). Baseline characteristics were analysed using frequency distribution and central tendency measurements; Mann-Whitney U-test (quantitative variables) and $\chi^{2}$ test (categorical variables) were used to estimate differences between sexes. Mean differences were tested using the paired sample t-test, which was used to compare metabolic variables across both initial measurements (IMs) and final measurements (FMs). Additionally, variables $\mathrm{BMI}, \mathrm{HbA1c}, \mathrm{SBP}, \mathrm{DBP}$, atherogenic index total cholesterol (TC) and/or high-density lipoprotein (HDL) (high risk of CVD $>4$ ), and atherogenic index plasma (high risk of CDV $>0.24$ ) were categorised and analysed by adjusting each of their means to the number of laboratory test results during the period of time in which the patient was actively receiving care through the programme, which only influenced the FM values. Furthermore, McNemar's tests were used to detect the differences in patients' IM and FM ECG, ABI, monofilament neuropathy test, ocular fundus, and categorised $\mathrm{HbA} 1 \mathrm{c}$.

Patients provided written informed consent before being enrolled. The study used a deidentified dataset to protect the confidentiality of the participants.

\section{Results}

The baseline demographic and clinical characteristics of the patients are shown in Table 1. The majority of the participants included were female ( $n=459,77.4 \%)$. Additionally, $82.1 \%$ ( $n=487$ ) of patients were on simvastatin therapy and $17.0 \%$ ( $n=101)$ were receiving gemfibrozil (data not shown). The annual attendance compliance ranged from $86.5 \%$ to $100 \%$. During the 10 years of follow-up, 64 participants died. Allcause mortality was $10.8 \%(95 \%$ confidence interval $[\mathrm{Cl}]=8.3$ to 13.3$)$ and cumulative incidence of CVD mortality was $2.7 \%(95 \% \mathrm{Cl}=1.4$ to 4.0$)$, representing $25.0 \%$ of all deaths.

All metabolic variables of interest are reported in Table 2, showing all IMs and FMs for average BMI, SBP, DBP, HbA1c, lipid profile, atherogenic indexes, urea, and creatinine during the follow-up period. There was an overall reduction of patient's weight; however, this loss was minimal and patients continued to be characterised as overweight. Although all final metabolic variable measurements, including $\mathrm{HbA} 1 \mathrm{c}$, showed mean differences comparing the IM and FMs, no significant differences in average SBP, urea, and creatinine were found. Regardless of the duration of illness, during follow-up no deterioration was determined in the renal function of patients.

Additionally, $54.5 \%$ of the patients' $\mathrm{HbA} 1 \mathrm{c}$ values were $<8.0 \%$ in their $\mathrm{IM}$; however, at the time of the FM, this percentage increased to $62.6 \%$ of the patients portraying $\mathrm{HbA} 1 \mathrm{c}$ values $<8.0 \%$. This shows a significant improvement $(P<0.001)$ in diabetes control as the number of patients with $\mathrm{HbA} 1 \mathrm{c}$ values of $<8.0 \%$ increased throughout the programme.

Figure 2 shows a comparison between the IMs and FMs of control by categorised metabolic variables. It was observed that the difference between categorised HbA1c groups, DBP ( $\geq 80 \mathrm{mmHg})$, atherogenic index (TC and/or HDL-cholesterol [c]) high-risk group, and atherogenic plasma index high-risk group were statistically significant $(P<0.001)$ among IMs and FMs. In groups categorised for 
Table 2 Mean difference between initial and final measurements for clinical features and metabolic variables in patients with type 2 diabetes mellitus.

\begin{tabular}{|c|c|c|c|c|c|c|}
\hline \multirow[b]{3}{*}{ Metabolic variables } & \multicolumn{4}{|c|}{ Measurements } & \multirow[b]{3}{*}{ Mean difference } & \multirow{3}{*}{$\begin{array}{c}P \text { - } \\
\text { value* }\end{array}$} \\
\hline & \multicolumn{2}{|c|}{$\begin{array}{c}\text { Initial, } \\
n=593\end{array}$} & \multicolumn{2}{|c|}{$\begin{array}{c}\text { Final, } \\
n=593\end{array}$} & & \\
\hline & Mean & SD & Mean & SD & & \\
\hline BMI & 29.32 & 4.94 & 29.06 & 4.61 & -0.26 & 0.013 \\
\hline SBP & 124.24 & 18.35 & 122.63 & 16.82 & -1.61 & 0.054 \\
\hline DBP & 75.22 & 11.08 & 71.84 & 9.13 & 3.37 & $<0.01$ \\
\hline $\mathrm{HbA1c}$ & 8.29 & 2.39 & 7.83 & 1.65 & 0.46 & $<0.01$ \\
\hline TC & 206.29 & 49.82 & 184.56 & 45.78 & 21.73 & $<0.01$ \\
\hline HDL-C & 54.96 & 17.59 & 59.63 & 14.73 & -4.67 & $<0.01$ \\
\hline LDL-C & 113.34 & 44.72 & 92.32 & 35.72 & 21.02 & $<0.01$ \\
\hline Triglycerides & 190.98 & 123.74 & 163.01 & 91.33 & 27.97 & $<0.01$ \\
\hline Urea & 40.10 & 15.82 & 41.16 & 23.81 & -1.05 & 0.272 \\
\hline Creatinine & 1.06 & 0.33 & 1.11 & 0.60 & -0.04 & 0.063 \\
\hline TC/HDL-C & 4.08 & 1.56 & 3.19 & 0.83 & 0.89 & $<0.01$ \\
\hline LDL/HDL-C & 2.29 & 1.23 & 1.60 & 0.64 & 0.69 & $<0.01$ \\
\hline TC-HDL-C & 151.32 & 49.44 & 124.92 & 41.78 & 26.40 & $<0.01$ \\
\hline TC-HDL-C/HDL-C & 3.08 & 1.56 & 2.19 & 0.83 & 0.89 & $<0.01$ \\
\hline TG/HDL-C & 3.83 & 2.63 & 2.95 & 2.19 & 0.88 & $<0.01$ \\
\hline AIP & 0.14 & 0.27 & 0.04 & 0.24 & 0.10 & $<0.01$ \\
\hline
\end{tabular}

$\mathrm{AIP}=$ atherogenic index plasma. $\mathrm{BMI}=$ body mass index. $\mathrm{DBP}=$ diastolic blood pressure. $\mathrm{HbA} 1 \mathrm{c}=$ glycated haemoglobin. $\mathrm{HDL}-\mathrm{C}=$ high-density lipoprotein cholesterol. $\mathrm{LDL}-\mathrm{C}=$ low-density lipoprotein cholesterol. SBP = systolic blood pressure. $\mathrm{SD}=$ standard deviation. $\mathrm{TC}=$ total cholesterol. $\mathrm{TG}=$ triglyceride.

*Paired samples t-test; statistically significant $P \leq 0.05$.

$\mathrm{HbA} 1 \mathrm{c}$, people with $\mathrm{HbA} 1 \mathrm{c}$ levels $>10.0 \%$ showed decreases of $3.6 \%$ ( $95 \% \mathrm{Cl}=3.5$ to 3.8 ) compared with the IM. On the other hand, people with $\mathrm{HbA} 1 \mathrm{c}<6.5 \%$ had a slight increase of $1.4 \%(95 \% \mathrm{Cl}=1.3$ to 1.6), with their $\mathrm{HbA} 1 \mathrm{c}$ value remaining within the adequate range for patients with T2DM.

According to the results summarised in Table 3, there was a statistically significant increase of $6.9 \%$ $(P<0.01)$ in the number of patients who had an abnormal ECG, from the IM $(59.5 \%)$ to the FM $(66.4 \%)$. The IM of monofilament neuropathy test increased significantly $(P<0.01)$ in the FM $(4.5 \%)$, suggesting a probable increasing relation with the years of duration of diabetes. The findings for $\mathrm{ABI}$ and ocular fundus were statistically non-significant.

\section{Discussion}

\section{Summary}

In this group of patients with T2DM, a comprehensive healthcare model was implemented. This was based on the original model proposed by the World Health Organization and the Pan American Health Organization. ${ }^{20}$ The MAIS for NCDs, with adjusted strategies to the care model, obtained favourable responses in participating patients over the 10 -year duration of the study.

\section{Strengths and limitations}

The success of the Chimbacalle diabetes programme is owing to many factors, but the most important one is the involvement of patients in their own health care. Each patient has their own printed record containing all of their laboratory, clinical, and anthropometric information; this strategy gives patients control over their own health and produces high attendance compliance. Furthermore, the feeling 


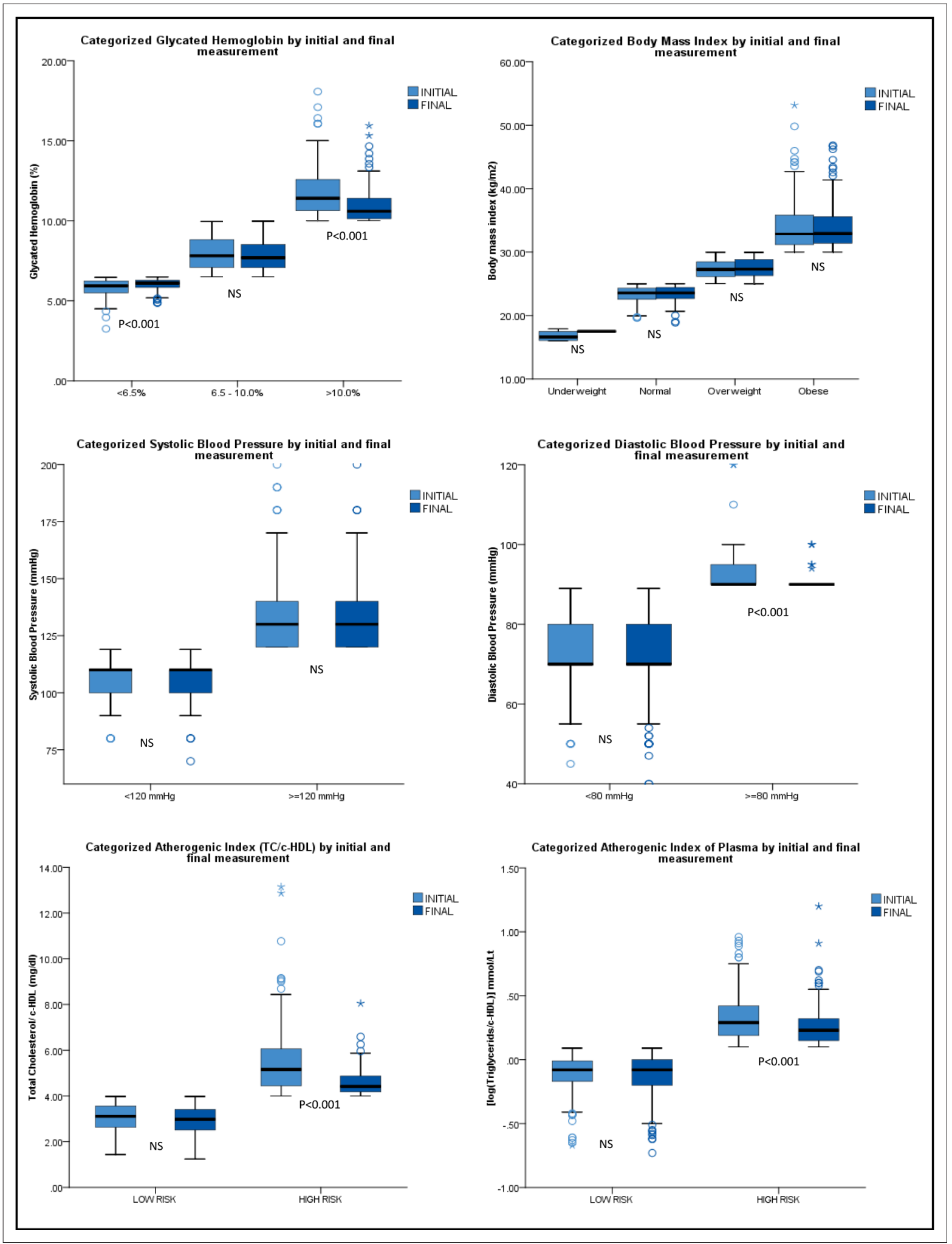

Figure 2 Metabolic variables categorised according to the initial and final measurement. Paired samples $t$-test; statistically significant $P \leq 0.05$. $H D L-C=$ high-density lipoprotein cholesterol. NS = non-significant. TC = total cholesterol. 
Table 3 Electrocardiogram, ankle-brachial index, monofilament neuropathy test, and ocular fundus by initial and final measurement in patients with type 2 diabetes mellitus.

\begin{tabular}{lccccc}
\hline & \multicolumn{5}{c}{ Measurement } \\
\hline
\end{tabular}

$\mathrm{ABI}=$ ankle-brachial index. $\mathrm{ECG}=$ electrocardiogram.

*Related-samples McNemar test; statistically significant $P \leq 0.05$

of belonging to a group of peers is transformed into motivation for the adherence to treatment, as well as in the prevention (patients become promoters of health within their family) and control of the disease. ${ }^{21}$

This study is a robust design, but its limitation was the loss of patients that could be a source of bias and a possible overvaluation of strength. Another limitation is the lack of sources of comparison. Given that almost every diagnosed patient in the centre joins the programme, the results cannot be compared with usual healthcare receivers in the same centre. Furthermore, the database of this study is quite unique and there is no similar information in other healthcare centres; however, this study introduces a protocol that can potentially improve patient management, and shows the excellent results of an integral diabetes programme with pharmacological and non-pharmacological intervention strategies that have contributed to public health.

\section{Comparison with existing literature}

This research uses an indicator of patient monitoring, metabolic control, and $\mathrm{HbA} 1 \mathrm{c}$. The study findings showed a decrease in $\mathrm{HbA} 1 \mathrm{c}$ from $8.3 \%$ to $7.8 \%$; it is important to consider that the applied model does not correspond to improved control. When HbA1c was reviewed and analysed in the ADVANCE, ACCORD, and VADT studies, reduction in $\mathrm{HbA} 1 \mathrm{c}$ with conventional therapy was $1.0 \%$, $0.6 \%$, and $1.0 \%$, respectively, which does not significantly differ from the population studied here. However, any reduction in HbA1c significantly reduces micro- and macrovascular complications. ${ }^{10,22,23}$

The general reduction of $0.5 \%$ in $\mathrm{HbA1c}$ found in this study was maintained during the 10-year follow-up, as found in the UK Prospective Diabetes Study (UKPDS), where a 1\% reduction in HbA1c was associated with a $37 \%$ decrease in microvascular risk. ${ }^{24}$ There are also some findings from Latin America, such as Chilean ${ }^{25}$ and Mexican ${ }^{26}$ studies, with a decrease of $0.4 \%-0.6 \%$ in the intervention group; $;^{27}$ and, Colombian ${ }^{28}$ and Peruvian ${ }^{29}$ studies showed a reduction of $1.3 \%$ up to $1.8 \%$ in the first year and no significant changes in the 4-year follow-up. Other studies around the world indicate that intervention reduces $\mathrm{Hb} 1 \mathrm{Ac}$ by about $0.46 \% .^{30}$

The prevalence of micro- and macrovascular complications in the study is lower than that reported in the literature, ${ }^{31}$ owing to early onset of pharmacological treatment with metformin at medium and high doses. ${ }^{32}$ Also, patients displaying inadequate response ( $\mathrm{HbA1c}>8.5 \%$ ) to oral hypoglycaemic drugs were started with Neutral Protamine Hagedorn (NPH) insulin therapy as soon as possible. 
Renoprotection using renin-angiotensin-aldosterone system blockers at minimum doses was given to patients with $\geq 5$ years of illness or when albuminuria is demonstrated. ${ }^{8,9}$ Furthermore, the stabilisation of lipid profiles took into account atherogenic-risk calculation and, therefore, the start of statins ${ }^{33}$ and the administration of acetylsalicylic acid (ASA) at low doses was based on the cardiovascular-risk score in people with diabetes. ${ }^{34,35}$ Even though there is controversy surrounding the daily use of ASA owing to the blood-thinning effect of aspirin, ${ }^{36}$ no side effects of bleeding have been recorded in these patients. Perhaps, the rate of complications and CVD mortality is extremely lower than other cohorts; ${ }^{37}$ further research is needed.

\section{Implications for practice}

Continuing education about diabetes is an essential component in prevention and treatment strategies. The educational strategy combined with clinical treatment provides the necessary incentives to face major changes in the patient's lifestyle. ${ }^{38}$ It is evident that the improvement in diabetes outcomes is linked to many factors; however, patient involvement in their own health care has been identified as a key factor in improving metabolic control of T2DM and delaying the appearance and progression of complications. ${ }^{39}$

An important aspect of this model of care is the permanence and continuity of the health personnel caring for patients. The same doctor and nurse have remained in the health team for 10 years. This is likely to have helped patients' engagement in the programme and the integration of the medical personnel in all patient contexts.

The initial diagnosis of diabetes is very stressful for patients. The initial shock and fear of this new diagnosis is a major barrier to understanding the indications given by the doctor. The healthcare staff help to resolve questions generated in the process of learning about the disease. Likewise, the staff create links between the medical team, the patient, and their family environment, creating a climate of trust that allows for better follow-up.

The country's health system guarantees the delivery of medication free of charge. It is observed that this could be considered a strategy in the appropriate metabolic control of patients. Adherence to treatment increases when distribution is free, and when laboratory and complementary exams are carried out continuously..$^{40}$ However, treatment is not sufficient without peer support and regular medical care.

In conclusion, Chimbacalle Medical Centre has successfully implemented a state-of-the-art programme that provides patients with T2DM with integral attention-focused care and a support network. By accurately complying with protocols, as well as international and national guidelines, the centre's programme has been useful in achieving adequate metabolic control and reduction of microand macrovascular complications. The unique nature of this programme has posed, to a certain extent, a limitation to this study, as a comparable database within the country is non-existent. However, the research presented has portrayed the achievement of positive outcomes, especially in the prevention of complications, despite the fact that the programme takes place in a primary healthcare centre that works with limited resources.

Funding

The authors declare that no funding from commercial or non-profit sectors was received for this study.

\section{Ethical approval}

This study was approved by the Institutional Review Board (IRB) of Central University of Ecuador (IRB ref number: 279-CE-UCE-2015)

\section{Provenance}

Freely submitted; externally peer reviewed.

\section{Acknowledgements}

The authors thank the staff and patients of Chimbacalle Medical Centre for their valuable contributions and trust through all these years. 


\section{References}

1. Tiwari R, Ghangale SS, lyer CM. Study of $\mathrm{HbA} 1 \mathrm{c}$ as a biomarker in dyslipidemia and atherogenicity in type 2 diabetes mellitus. International Journal of Clinical and Biomedical Research 2015; 1(2): 5-11.

2. International Diabetes Federation. IDF diabetes atlas. Ninth edition 2019. 2019; https://diabetesatlas.org/upload/ resources/material/20200302_133351_IDFATLAS9e-final-web.pdf (accessed 2 Apr 2020).

3. International Diabetes Federation. IDF diabetes atlas. Eighth edition 2017. 2017; https://www.idf.org/e-library/ epidemiology-research/diabetes-atlas/134-idf-diabetes-atlas-8th-edition.html (accessed 2 Apr 2020).

4. Ministerio de Salud Publico del Ecuador. Manual del Modelo de Antencion Integral de Salud — MAIS. 2012; http:// instituciones.msp.gob.ec/somossalud/images/documentos/guia/Manual_MAIS-MSP12.12.12.pdf (accessed 8 Apr 2020).

5. García R, Suárez R. [Education for people with diabetes mellitus in primary health care]. [Article in Spanish]. Revista Cubana de Endocrinología 2007; 18(1)

6. Kunutsor SK, Seidu S, Khunti K. Aspirin for primary prevention of cardiovascular and all-cause mortality events in diabetes: updated meta-analysis of randomized controlled trials. Diabet Med 2017; 34(3): 316-327. DOI: https:// doi.org/10.1111/dme.13133

7. Antithrombotic Trialists' Collaboration. Collaborative meta-analysis of randomised trials of antiplatelet therapy for prevention of death, myocardial infarction, and stroke in high risk patients. BMJ 2002; 324(7329): 71-86. DOI: https://doi.org/10.1136/bmj.324.7329.71

8. Barnett $\mathrm{AH}$, Bain SC, Bouter $\mathrm{P}$, et al. Angiotensin-receptor blockade versus converting-enzyme inhibition in type 2 diabetes and nephropathy. N Engl J Med 2004; 351(19): 1952-1961. DOI: https://doi.org/10.1056/NEJMoa042274

9. Brenner BM, Cooper ME, de Zeeuw D, et al. Effects of losartan on renal and cardiovascular outcomes in patients with type 2 diabetes and nephropathy. N Engl J Med 2001; 345(12): 861-869. DOI: https://doi.org/10.1056/ NEJMoa011161

10. Ginsberg HN, Elam MB, Lovato LC, et al. Accord Study Group. Effects of combination lipid therapy in type 2 diabetes mellitus. N Engl J Med 2010; 362(17): 1563-1574. DOI: https://doi.org/10.1056/NEJMoa1001282

11. Taylor F, Huffman MD, Macedo AF, et al. Statins for the primary prevention of cardiovascular disease. Cochrane Database Syst Rev 2013; 2013(1): CD004816.

12. Holman RR, Paul SK, Bethel MA, et al. 10-year follow-up of intensive glucose control in type 2 diabetes. N Engl J Med 2008; 359(15): 1577-1589. DOI: https://doi.org/10.1056/NEJMoa0806470

13. Cryer DR, Nicholas SP, Henry DH, et al. Comparative outcomes study of metformin intervention versus conventional approach the COSMIC approach study. Diabetes Care 2005; 28(3): 539-543. DOI: https://doi.org/10. 2337/diacare.28.3.539

14. Frid A, Hirsch L, Gaspar R, et al. New injection recommendations for patients with diabetes. Diabetes Metab 2010; 36 Suppl 2: S3-S18. DOI: https://doi.org/10.1016/S1262-3636(10)70002-1

15. Vaag A, Lund SS.Insulin initiation in patients with type 2 diabetes mellitus: treatment guidelines, clinical evidence and patterns of use of basal vs premixed insulin analogues. Eur J Endocrinol 2012; 166(2): 159-170. DOI: https:// doi.org/10.1530/EJE-11-0022

16. Ministry of Public Health of Ecuador. Clinical practice guide (CpG) for type 2 diabetes mellitus. 2017; https://www. salud.gob.ec/wp-content/uploads/downloads/2017/05/Diabetes-mellitus_GPC.pdf (accessed 8 Apr 2020).

17. Crespo I, Calderón R, Delgado F, et al. [Peruvian guide for diagnosis, control and treatment of type 2 diabetes mellitus] Guía peruana de diagnóstico,control y tratamiento de ladiabetes mellitus tipo 2. 2008; http://www. endocrinoperu.org/sites/default/files/Guia\%20Peruana\%20de\%20Diagn\%C3\%B3stico\%20\%20Control\%20y\%20\% 20Tratamiento\%20de\%20la\%20Diabetes\%20Mellitus\%202008.pdf (accessed 8 Apr 2020).

18. Aryal M, Poudel A, Satyal B, et al. Evaluation of non-HDL-c and total cholesterol: HDL-C ratio as cumulative marker of cardiovascular risk in diabetes mellitus. Kathmandu Univ Med J 2010; 8(32): 398-404. DOI: https://doi.org/10. 3126/kumj.v8i4.6239

19. Gasevic D, Frohlich J, Mancini GJ, et al. Clinical usefulness of lipid ratios to identify men and women with metabolic syndrome: a cross-sectional study. Lipids Health Dis 2014; 13: 159. DOI: https://doi.org/10.1186/1476511X-13-159

20. Pan American Health Organization, World Health Organization. Innovative care for chronic conditions: organizing and delivering high quality care for chronic noncommunicable diseases in the Americas. 2013; https://www.paho. org/hq/dmdocuments/2013/PAHO-Innovate-Care-2013-Eng.pdf (accessed 2 Apr 2020).

21. Rodríguez-Campuzano MDL, Rentería-Rodríguez A, Rosales-Arellano A, et al. [Educational proposal for the health of patients with type 2 diabetes mellitus]. [Article in Spanish]. Pensando Psicología 2014; 10(17): 103-112.

22. Patel A, MacMahon S, Chalmers J, et al. Advance Collaborative Group. Intensive blood glucose control and vascular outcomes in patients with type 2 diabetes. N Engl J Med 2008; 358(24): 2560-2572. DOI: https://doi.org/ 10.1056/NEJMoa0802987

23. Duckworth W, Abraira C, Moritz T, et al. VADT Investigators. Glucose control and vascular complications in veterans with type 2 diabetes. N Engl J Med 2009; 360(2): 129-139. DOI: https://doi.org/10.1056/NEJMoa0808431

24. Stratton IM, Adler Al, Neil HA, et al. Association of glycaemia with macrovascular and microvascular complications of type 2 diabetes (UKPDS 35): prospective observational study. BMJ 2000; 321(7258): 405-412. DOI: https://doi. org/10.1136/bmj.321.7258.405

25. Bächler R, Mujica V, Orellana C, et al. Eficacia de un programa educativo estructurado en población diabética chilena [Effectiveness of a structured educative program in Chilean diabetic patients]. Revista médica de Chile 2017; 145(2): 181-187. DOI: https://doi.org/10.4067/S0034-98872017000200005 
26. Muñoz-Torres AV, García-Peña C, García-Peña C, et al. Fiber in diet is associated with improvement of glycated hemoglobin and lipid profile in Mexican patients with type 2 diabetes. J Diabetes Res 2016; 2016: 1-9.

27. Gagliardino JJ, de la Hera M, Siri F, Grupo de Investigación de la Red QUALIDIAB. [Evaluation of the quality of care for diabetic patients in Latin America]. [Article in Spanish]. Rev Panam Salud Publica 2001; 10(5): 309-317. DOI: https://doi.org/10.1590/S1020-49892001001100003

28. Kattah W, Coral P, Méndez F. [Assessment of a treatment and education program in reducing the glucosilated hemoglobine levels in diabetic patients]. [Article in Spanish]. Acta Medica Colomb 2007; 32(4): $206-211$.

29. Guzmán-Priego CG, Baeza-Flores G del C, Atilano-Jiménez D, et al. [Effect of an educational intervention on biochemical parameters of diabetic patients in an institutional health service]. [Article in Spanish]. Atención Familiar 2017; 24(2): 82-86.

30. Lalić NM, Lalić K, Jotić A, et al. The impact of structured self-monitoring of blood glucose combined with intensive education on $\mathrm{HbA} 1 \mathrm{c}$ levels, hospitalizations, and quality-of-life parameters in insulin-treated patients with diabetes at primary care in Serbia: the multicenter SPA-EDU study. J Diabetes Sci Technol 2017; 11(4): 746-752. DOI: https://doi.org/10.1177/1932296816681323

31. Morgan CLI, Owens DR, Aubonnet $P$, et al. Primary prevention of diabetic retinopathy with fibrates: a retrospective, matched cohort study. BMJ Open 2013; 3(12): e004025. DOI: https://doi.org/10.1136/bmjopen-2013-004025

32. Petrovick GF. Type 2 diabetes mellitus and metformin hydrochloride usage: a short review. J Pharmacol Ther Res 2018; 2(2): 6-9. DOI: https://doi.org/10.35841/pharmacology.2.2.6-9

33. Ramos PM. [Statins: from high potency to extralipid effects]. [Article in Spanish]. Rev Esp Cardiol 2015; 15(Supp 1): 22-27.

34. American Diabetes Association. Standards of medical care in diabetes - 2017. Diabetes Care 2017; 40(Suppl 1): $1-142$.

35. Stevens RJ, Kothari V, Adler Al, et al. The UKPDS risk engine: a model for the risk of coronary heart disease in type Il diabetes (UKPDS 56. Clin Sci 2001; 101(6): 671-679. DOI: https://doi.org/10.1042/cs1010671

36. Arnett DK, Blumenthal RS, Albert MA, et al. 2019 ACC/AHA guideline on the primary prevention of cardiovascular disease: a report of the American College of Cardiology/American Heart Association Task Force on Clinical Practice Guidelines. Circulation 2019; 140(11): e596-e646. DOI: https://doi.org/10.1161/CIR.0000000000000678

37. Einarson TR, Acs A, Ludwig C, et al. Prevalence of cardiovascular disease in type 2 diabetes: a systematic literature review of scientific evidence from across the world in 2007-2017. Cardiovasc Diabetol 2018; 17(1): 83. DOI: https:// doi.org/10.1186/s12933-018-0728-6

38. Urbán-Reyes BR, Coghlan-López JJ, Castañeda-Sánchez O. [Lifestyle and glycemic control in patients diagnosed with diabetes mellitus in the primary care level]. [Article in Spanish]. Atención Familiar 2015; 22(3): 68-71.

39. Salci MA, Meirelles BHS, da Silva DMGV.Primary care for diabetes mellitus patients from the perspective of the care model for chronic conditions. Rev Lat Am Enfermagem 2017; 25: e2882. DOI: https://doi.org/10.1590/15188345.1474.2882

40. Peper FE, Esteban S, Terrasa SA. [Evaluation of primary adherence to medications in patients with chronic conditions: a retrospective cohort study]. [Article in Spanish]. Aten Primaria 2018; 50(2): 96-105. DOI: https://doi. org/10.1016/j.aprim.2017.01.013 